\title{
Battery Assignment and Scheduling for Drone Delivery Businesses
}

\author{
Sangyoung Park, Licong Zhang, and Samarjit Chakraborty \\ Chair of Real-Time Computer Systems, Technical University of Munich, Germany \\ \{park, licong.zhang\}@ rcs.ei.tum.de, samarjit@tum.de
}

\begin{abstract}
Recent advances in battery and drone technologies have opened up possibilities of commercial use of drones. Private companies are looking into the possibilities of using drones for commercial deliveries from the legal, technical, and economical perspective. Nevertheless, the battery management perspective of such businesses has not yet been thoroughly investigated. In this paper, we identify that battery management of such application has a major impact of the costs, and formulate an optimization problem to reduce the aging of batteries. We identify two subproblems, battery assignment, and battery scheduling to derive a solution that minimizes the aging of the batteries. We show that the formulation enables leveraging the trade-off relationships between the packet waiting time and battery purchasing cost. The experimental results show the proposed method reduce the electricity and battery purchasing cost by $25 \%$, and average packet waiting time by more than $50 \%$.
\end{abstract}

\section{INTRODUCTION}

Drone delivery business is a promising application of unmanned aerial vehicles (UAV). Unlike their traditional applications, which was confined to military surveillance or tactical use, companies in private sector are investigating the potential of drones delivery business in various aspects. For example, Amazon Prime Air is targeting to deliver products to doorsteps of customers within 30 minutes of ordering [3]. It targets packages weighing less than 5 pounds, with a delivery distance of less 10 miles. DHL's parcelcopter has shown its capability of delivering products to remote locations [1]. While many enterprises are pushing forward to overcome the legal and technical hurdles for such businesses, little has been done from the research community on addressing technical hurdles from the battery management perspective.

Battery management will be one of the major factors affecting the time-to-delivery and profitability of the business. Fig. 1 shows the battery management of a drone delivery business. A set of available batteries, likely to comprise heterogeneous packs, serves a set of incoming delivery tasks as the customers make orders online. The batteries can be attached and detached from the drones in order to maximize the utilization of both the batteries and the drones as some commercial enterprises realized the concept [4], [2]. It avoids long wait times of drones for their batteries to be charged after every flight, which will be longer than the flight times themselves. After a delivery is made, batteries are recharged for next use. Meanwhile, the state-of-health ( $\mathrm{SOH})$ of batteries degrades due to cycle aging and calendar aging resulting in reduced battery capacity. If their capacity reduces to a certain threshold, i.e., $80 \%$, they are no longer fit for service, and are retired to be replaced by equivalent number of new batteries.

The $\mathrm{SOH}$ degradation of batteries will be a particularly important issue even when compared with other batterypowered applications, e.g., electric vehicles (EV). The delivery

978-1-5090-6023-8/17/\$31.00 @2017 IEEE

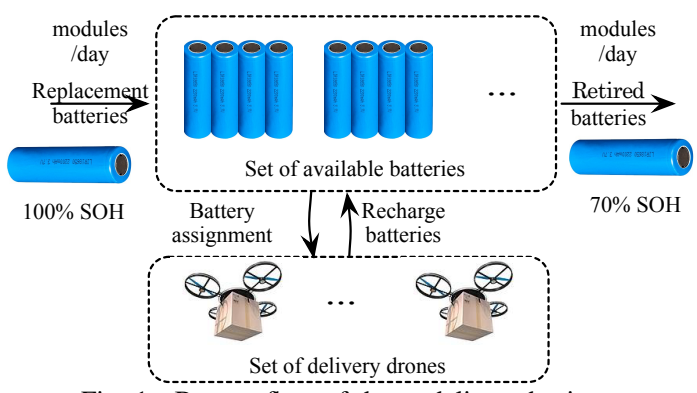

Fig. 1. Battery flow of drone delivery business.

drones are even more weight-sensitive and operates under a harsher condition that EVs. The minimum amount of battery should be carried by the drones to avoid the snowball effect. The more battery weight it carries, the more energy it requires, and hence again requiring additional battery weight. Moreover, most of the delivery drones are targeting to deliver a product within 30 minutes mandating the batteries to be discharged at a rate from well over $2 \mathrm{C}$, much higher than typical currents seen in an electric vehicle. This all leads to exploitation of the batteries that accelerates the $\mathrm{SOH}$ degradation of the batteries.

Despite recent price reduction of $\mathrm{Li}$-ion batteries, probably the only option for battery-powered drones, their price is still quite high. Our simulation results show that with an electricity price of 20 cents/kWh and the Li-ion battery price of $500 \mathrm{USD} / \mathrm{kWh}$, the recurring battery purchasing cost would be around $60 \%$ of the electricity cost [13]. Therefore, it is important to tackle $\mathrm{SOH}$ degradation by managing batteries in an optimized fashion. In this paper, we identify two subproblems for the battery management, battery assignment and battery scheduling, under the objective of minimizing the battery $\mathrm{SOH}$ degradation. As numerous prior works attest to, two major factors that affect the rate of $\mathrm{SOH}$ degradation are the average state-of-charge (SOC) and SOC swing of the batteries [10], [15], [14]. The larger the average SOC and the SOC swing, the faster SOH degradation. Hence, we propose a solution framework that reduces the average SOC and the SOC swing.

The contributions of this paper are summarized as follows.

- We, for the first time, formulate the battery assignment and battery scheduling problem under the objective of minimizing the $\mathrm{SOH}$ degradation of the whole battery set.

- The solution is divided into two stages where the battery assignment is solved by a heuristic algorithm, and battery scheduling is solved by an mixed integer linear programming (ILP).

- We show that our formulation is capable of leveraging the trade-off relationship between $\mathrm{SOH}$ degradation and 


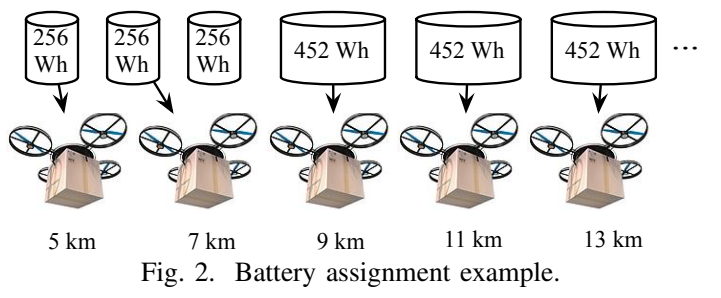

packet waiting time.

- The solution scheme is integrated with an discrete event simulator that features elaborate equivalent circuit battery models and aging parameters to verify the results.

The experimental results show the proposed method reduce the electricity and battery purchasing cost by $25 \%$, and average packet waiting time by more than $50 \%$.

\section{RELATED WORK}

Recently, a number of e-commerce and logistics companies began looking into possibility of drones for product deliveries. DHL has implemented its own delivery drone and successfully delivered products in remote locations [1]. E-commerce giant Amazon has been developing a new service, Amazon Prime Air, since 2013, in order to deliver products within 30 minutes of ordering [3]. There exist numerous prior works on autonomous navigation and control of drones from the research community as well. Autonomous navigation and control of drones are crucial for realizing such services [5], [8]. An early framework has been proposed for autonomous drone delivery including loading and release mechanisms of packages without human intervention, autopilot software, and communication infrastructure to achieve autonomous package delivery by drones [9]. [16] demonstrated a automatic battery replacement platform, which attaches and detaches batteries to/from drones without human intervention. This avoids drones waiting for lengthy battery charge times and enables maximizing the utilization of drones. A similar concept of battery swapping has been proposed by Tesla Motors [11]. All the above mentioned works indicate there is a large body of research from both industry and academia working toward realizing an autonomous framework for drone delivery services.

Nevertheless, all the works rather focused on technical hurdles on individual drones, and there has not been much work focusing on the battery management for the whole delivery service. [7] addresses some important challenges for the drone delivery service, vehicle design, navigation, coordination between drones, and electricity and battery cost. However, the work merely introduces these challenges and much of the research questions are left open. Regarding the holistic analysis of similar applications involving many vehicles and batteries, an economic analysis on a taxi fleet with battery swapping stations has been performed [12]. However, this work lacks details on battery models, and hence, is inept at evaluating techniques that considers complex battery characteristics such as aging according to battery usage patterns. In comparison, in our work, we can test and evaluate different management policies using the proposed simulator. A recent work of ours has performed preliminary economic analysis on drone delivery businesses taking into account the battery set composition, battery aging, and so on, on electricity cost, battery purchasing cost and time-to-delivery of the packages [13].

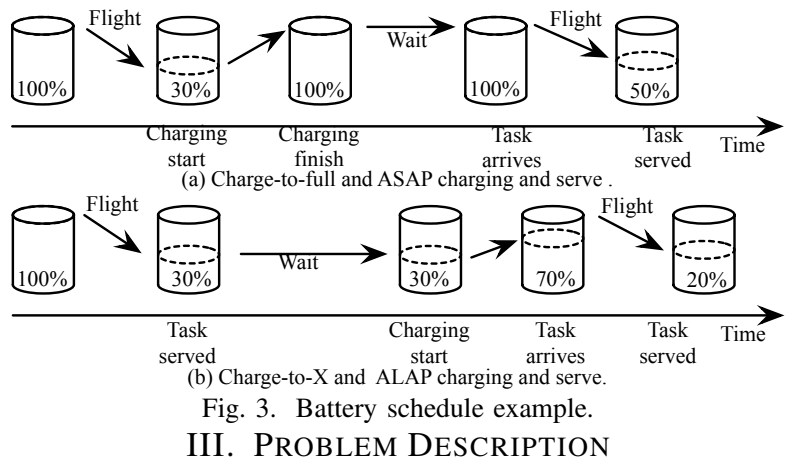

In this section, we formulate the battery management problem for the drone delivery business. Before moving on to the mathematical formulation, we first list some underlying assumptions and show how the problem could influence the objectives for this business. The first assumption is that batteries can be attached and detached from the drones as discussed in Section I. This helps maximizing the drone utilization as it would not have to wait for its batteries to charge. Secondly, we assume that heterogeneous sizes of batteries are used for drone delivery. The distance to destination may differ greatly among orders, and hence, the energy requirements. Therefore, attaching smaller batteries to deliveries over a small distance could reduce the energy consumption.

The battery management problem involves two interconnected sub-problems, namely the battery assignment and the battery scheduling problem. The former refers to the problem of mapping a set of batteries onto the delivery services. The battery assignment problem finds an assignment that is the most beneficial for mitigating the $\mathrm{SOH}$ degradation of the whole battery set. Fig. 2 depicts a battery assignment. The figure depicts usage of two $256 \mathrm{Wh}$ batteries and three $452 \mathrm{Wh}$ batteries. However, other options are available such as usage of three $256 \mathrm{Wh}$ batteries to serve $5 \mathrm{~km}, 7 \mathrm{~km}, 9 \mathrm{~km}$ deliveries and two $452 \mathrm{Wh}$ batteries for the rest. The second solution allows drones to carry less battery weight leading to less energy consumption as well as mitigating the $\mathrm{SOH}$ degradation for overall battery set. Therefore, in this paper, we assume that a task will be assigned a battery with the appropriate capacity, i.e., a task will not use a battery with a larger capacity if one with smaller capacity will suffice.

The scheduling problem refers to the problem determining the time instances at which a battery begins charging, terminates charging, begins discharging, and terminates discharging. Fig. 3 depicts two different battery schedules, which serves two identical delivery services. Each timeline refers to a schedule that one battery goes through a time period. Fig. 3(a) shows a schedule where a battery is charged immediately to the full capacity after a delivery service while the schedule in (b) delays charging and does not charge the battery to its full capacity. Schedule shown in (b) reduces the average SOC of the battery, which is known to have mitigating effect on $\mathrm{SOH}$ degradation as will be shown in the next section. In this paper, we utilize battery assignment and scheduling as the two control knobs in mitigating the $\mathrm{SOH}$ degradation of the whole battery set as will be shown in the subsequent sections.

To formulate the problem mathematically, we first define a set of tasks, which denote the delivery tasks of the drones. A task $t_{i} \in T$ can be characterized as $t_{i} \sim\left\{a_{i}^{t}, o_{i}^{t}, e_{i}, l_{i}^{t}, b_{i}\right\}$, where $a_{i}^{t}, o_{i}^{t}, e_{i}, l_{i}^{t}, b_{i}$ represent respectively the arrival time, 


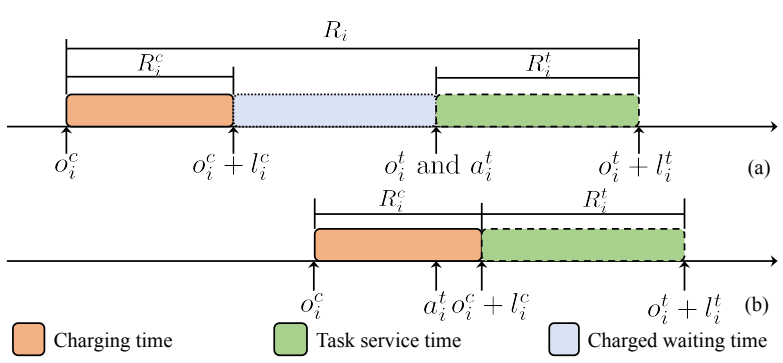

Fig. 4. Two example service schedules. (a) The battery is charged and waits until the task is arrived and immediately dispatched. (b) The task arrives and waits until the charging operation is finished to be dispatched.

dispatch time, energy required, flight time and ID of the battery assigned to this task. Since we assume heterogeneous types of batteries, $e_{i}$ should differ for different types of batteries. We further define a set of charging operations $c_{i} \in C$ as a tuple $c_{i} \sim\left\{o_{i}^{c}, e_{i}, l_{i}^{c}, b_{i}, c h_{i}\right\}$, where $o_{i}^{c}, e_{i}, l_{i}^{c}, b_{i}, c h_{i}$ denote respectively the start time for charging, energy to charge, length of charging, battery ID and charger ID. Furthermore, we define a set of batteries $b_{i} \in B$ and chargers $c h_{i} \in C H$.

For each battery, a task is always preceded by a charging operation. In the case of no charging operation is required before a task (e.g., because there is sufficient charge left in the battery), we consider a charging operation of zero time $\left(l_{i}^{c}=0\right)$. Thus, we define a set of services as a pair of charging operation and task $s_{i} \in S$. Then a service can be represented as a tuple $s_{i} \sim\left\{a_{i}^{t}, o_{i}^{t}, l_{i}^{t}, o_{i}^{c}, l_{i}^{c}, e_{i}, b_{i}, c h_{i}\right\}$. In addition, we define three time regions for each service: (i) the charging region as $R_{i}^{c}=\left[o_{i}^{c}, o_{i}^{c}+l_{i}^{c}\right]$, (ii) the task region as $R_{i}^{t}=\left[o_{i}^{t}, o_{i}^{t}+l_{i}^{t}\right]$ and (iii) the service region as $R_{i}=\left[o_{i}^{c}, o_{i}^{t}+l_{i}^{t}\right]$. An example of a service and the three regions is shown in Fig. 4.

Here, we divide the variables into two categories. The variables that are given include $a_{i}^{t}, l_{i}^{t}$ and $e_{i}$, which are specified by the task profile. The variables that need to be determined include $o_{i}^{t}, o_{i}^{c}, l_{i}^{c}, b_{i}, c h_{i}$. The assignment problem is to determine for each $s_{i}$ the battery $b_{i}$ and the charging post $c h_{i}$ involved. The scheduling problem is to determine the schedule for charging, the length of charging and the schedule for task serving, namely $o_{i}^{c}, l_{i}^{c}$ and $o_{i}^{t}$.

In general, we are interested in two objectives. The first objective to reduce the average waiting time of tasks, i.e., a delivery task can be carried out as soon as possible once it has arrived. This is crucial for drone delivery services as time-todelivery is a critical measure of such businesses. The second objective is to reduce the $\mathrm{SOH}$ degradation of the batteries. The objectives will be elaborated in Section V.

\section{BATTERY SOH DEGRADATION}

$\mathrm{SOH}$ degradation refers to deterioration of battery conditions in the form of impedance increase, voltage decrease, and most importantly, capacity fading. The model from [10] describes capacity fading. Average SOC, $\overline{S O C}$, and standard deviation of SOC, $\sigma_{S O C}$, and the operating temperature, $T_{B}$, are the main factors affecting the rate of capacity fading. The model calculates essentially the two intermediate parameters, $L_{1}$, and $L_{2}$, in order to calculate the aging factor, $L$.

$$
\begin{gathered}
L_{1}=K_{c o} N \cdot \exp \left(\left(\sigma_{S O C}-1\right) \frac{T_{r e f}+273}{K_{e x}\left(T_{B}+273\right)}\right)+0.2 \frac{t_{\text {cycle }}}{t_{\text {life }}}, \\
L_{2}=L_{1} \exp \left(4 K_{\text {soc }}(\overline{S O C}-0.5)\right)(1-L),
\end{gathered}
$$
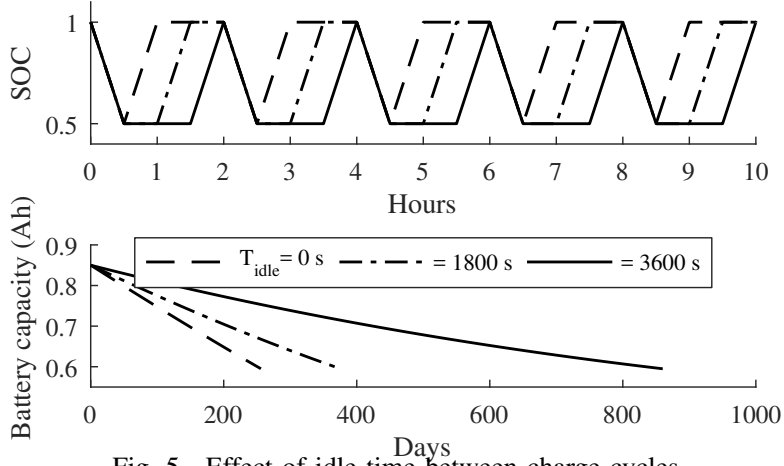

Fig. 5. Effect of idle time between charge cycles.

where $K_{c o}, K_{e x}, K_{S O C}$ are battery specific empirical constants where the values are given in [10] for an A123 ANR26650M1A cell. $T_{r e f}$ is the reference battery temperature of $25^{\circ} \mathrm{C}$. $t_{\text {cycle }}$ is the duration of one cycle and $t_{\text {life }}$ the shelf life at $25^{\circ} \mathrm{C}$ at $50 \%$ SOC until $80 \%$ of the initial capacity remain. Parameter $L_{1}$ reflects the effect of SOC deviation while parameter $L_{2}$ reflects the effect of average SOC on SOH degradation. The total increase in the life parameter is

$$
L\left(T_{m}\right)=L_{2} \exp \left(K_{t}\left(T_{B}-T_{\text {ref }}\right) \frac{T_{r e f}+273}{T_{B}+273}\right),
$$

where $K_{t}$ accounts for a doubling of the decay rate for each $10^{\circ} \mathrm{C}$ rise in temperature.

We have integrated the $\mathrm{SOH}$ model to the Li-ion battery equivalent circuit model given in [6] to construct a battery model capable of simulating both the short-term efficiency as well as the long-term SOH degradation. Using the model, we have done some preliminary simulations on battery aging resembling our problem setup. Fig. 5 shows the simulation result on how the idle time between a discharge and a charge operation affect the $\mathrm{SOH}$ degradation. Battery cells go through multiple cycles with a period of two hours differing in idle period $T_{i d l e}$ as show in the upper figure. Delaying the charging operation as much as possible reduces the average SOC significantly which mitigates the capacity fading. Fig. 6 shows

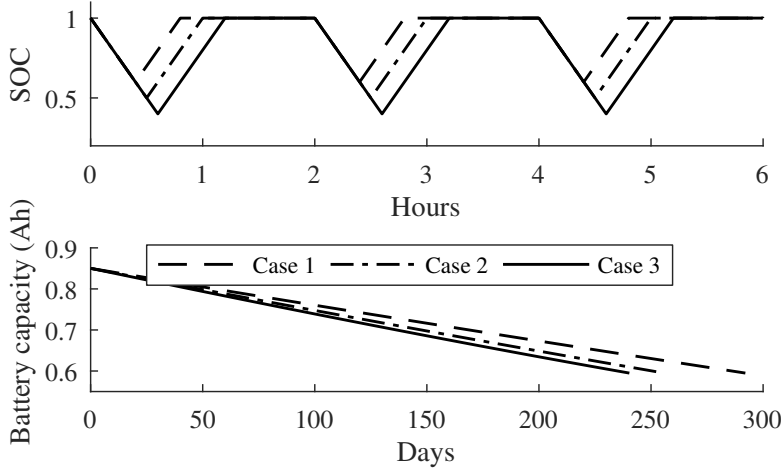

Fig. 6. Effect of DoD on aging.

another simulation result where the depth of discharge (DoD) differs. The larger the DoD is, the larger the capacity fading becomes. Poor battery assignment results in carrying additional battery weight, which increases the energy consumption and average DoD of the battery set. We emphasize that these observations are not limited to the $\mathrm{SOH}$ model [10], but are in line prior observations in the literature [15], [14]. We take a cue from these observations and formulate an optimization problem to minimize the $\mathrm{SOH}$ degradation of the batteries in a drone delivery services in the following section. 


\section{BATTERy ASSIGNMENT AND SCHEDULING ALGORITHMS}

The battery management problem can be formulated as a constraint-driven optimization problem. However, the combined problem suffers from a large complexity and the problem easily becomes intractable as the size becomes large. In order to tackle the large complexity, we divide the problem into two parts, namely (i) the battery and charger assignment problem, where the batteries and chargers are assigned to the services with a temporal order, and (ii) the scheduling problem, where the schedules of charging and task dispatching are computed.

\section{A. Battery Assignment}

The objective of the assignment problem is to (i) map the batteries to the corresponding services, i.e., to determine $b_{i}$ for the service $s_{i}$ and (ii) the batteries to the chargers, i.e., to determine $c h_{i}$ for the service $s_{i}$. The temporal order of the services mapped on the batteries and chargers is also determined. Here, we use a greedy heuristic for both problems, where the batteries and chargers are assigned to services in a greedy fashion according to the arrival time of the tasks. We assume in the assignment and scheduling problem, that the charging and discharging of the batteries are of constant rate, where $k^{c}$ and $k^{t}$ denote the charging and discharging rate of the battery. Algorithm 1 shows the how the batteries are assigned. Line 1-2 divides the batteries and services into types according to the battery capacity and the required energy of tasks. In Line 3-23, each battery type is gone through for the battery assignment. In Line 4, each type of services are sorted in ascending order according to an estimated time for the start of the charging operation, which can be denoted as $a_{i}^{t}-e_{i} / k^{c}$, where $e_{i}$ denotes the energy required by the service for the chosen battery type. Line 5-11 appends attributes $t_{e a}, S$ and order to each battery, which denote respectively the earliest available time of the battery, the services mapped to the battery and the temporal order. Line 12-19 assigns the services to the batteries. Line 13 finds the earliest estimated services that is not assigned and Line 14 finds the battery that will become available first according to estimation. Line 16 updates the current battery with the next estimated available time, which is the maximal value of $t_{e a}+e_{i} / k^{t}+e_{i} / k^{c}$ and $a_{i}^{t}+e_{i} / k^{t}$. Line 15 and 18 assign the battery to the corresponding service and vise versa. The chargers can then be assigned to the services in a similar manner.

\section{B. Battery Scheduling}

Once the batteries and chargers are assigned to the services, we formulate the scheduling problem into an ILP problem. The constraints for the scheduling problem are listed as follows.

(C1) Charge-and-serve constraint: This constraint enforces that the tasks are dispatched only when the charging operation is finished.

$$
\forall s_{i} \in S, o_{i}^{c}+l_{i}^{c} \leq o_{i}^{t}
$$

(C2) Arrive-and-serve constraint: This constraint enforces that the tasks are dispatched only after the arrival time.

$$
\forall s_{i} \in S, a_{i}^{t} \leq o_{i}^{t}
$$

(C3) Non-overlapping service constraint: A battery can only be utilized by one service simultaneously.

$$
\forall b_{j} \in B, \forall s_{i} \in b_{j} . S, o_{i}^{t}+l_{i}^{t} \leq o_{i+1}^{c}
$$

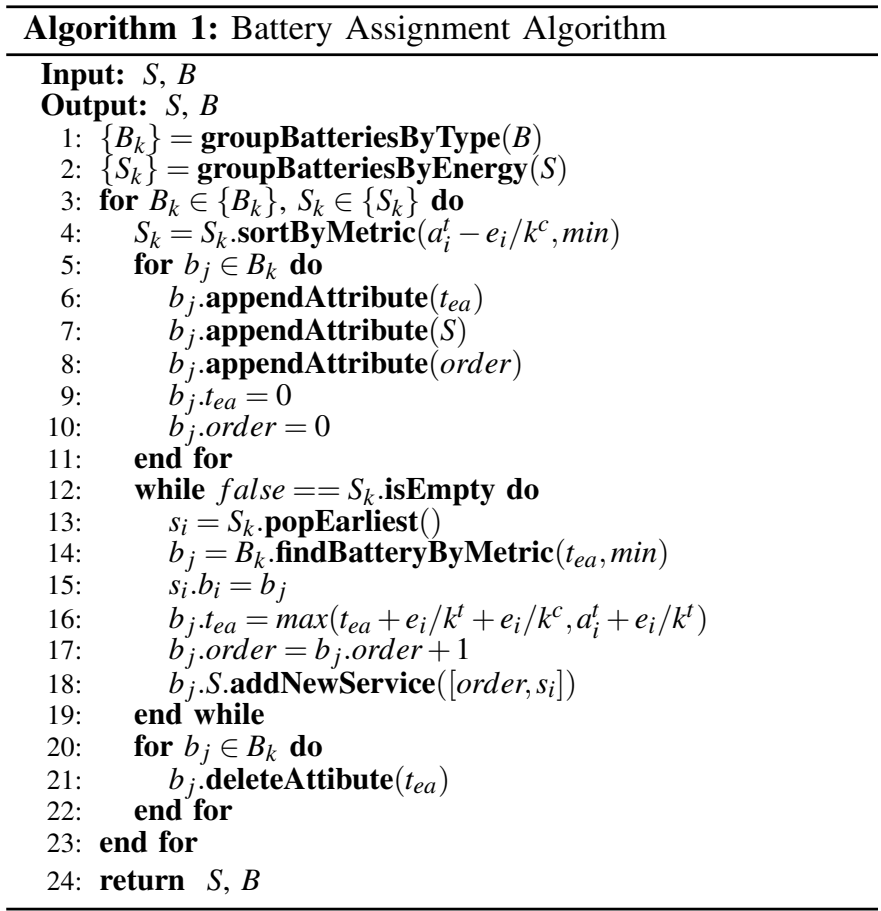

(C4) Non-overlapping charging operation constraint: Similarly, a charger can only be utilized by one service.

$$
\forall c h_{j} \in C H, \forall s_{i} \in c h_{j} . S, o_{i}^{c}+l_{i}^{c} \leq o_{i+1}^{c}
$$

(C5) Sufficient charge constraint: This constraint enforces that in a service, the battery always has sufficient charge for the task. $\forall s_{m} \in E\left(s_{i}, b_{j}\right)$ denote here all the services that use the same battery $b_{j}$ and are scheduled earlier than $s_{i}$.

$$
\forall b_{j} \in B, \forall s_{i} \in b_{j} . S, \sum_{\forall s_{m} \in E\left(s_{i}, b_{j}\right)}\left(l_{m}^{c} k^{c}-l_{m}^{t} k^{t}\right)+l_{i}^{c} k^{c}-l_{i}^{t} k^{t} \geq 0
$$

(C6) Battery charge capacity constraint: The battery can not be charged beyond its current maximal capacity. Here, $b_{j}$.cap denotes the maximal capacity of battery $b_{j}$. In a single scheduling period (e.g. one day), we consider it to be constant. This value, however, is expected to decrease in the long term, as discussed in Section IV.

$$
\forall b_{j} \in B, \forall s_{i} \in b_{j} . S, \sum_{\forall s_{m} \in E\left(s_{i}, b_{j}\right)}\left(l_{m}^{c} k^{c}-l_{m}^{t} k^{t}\right)+l_{i}^{c} k^{c} \leq b_{j} . c a p
$$

The objectives we consider is on one hand the average task waiting time and the $\mathrm{SOH}$ degradation on the other. We have shown in Section IV that one important factor for the $\mathrm{SOH}$ degradation rate is the average SOC of the batteries. We, therefore, use two linear objectives to represent the $\mathrm{SOH}$ degradation, namely the average left-over charge and the average charged waiting time, which have a direct impact on the average SOC. Thus, we consider three objectives in this scheduling problem, which can be formulated as follows. We use a weighted sum of the objectives as the final objective of the problem. The weight combinations can explored to achieve the design requirements. The scheduling problem can then be formulated into an ILP problem and solved by an external solver. 


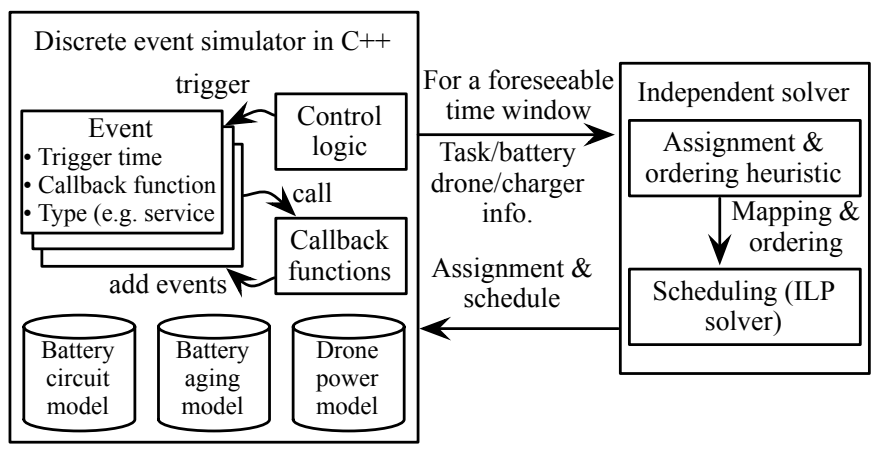

Fig. 7. Discrete event simulator for drone delivery integrated with proposed solution methodology.

(O1) Average task waiting time: This objective describes the average waiting time of the tasks, which is represented as the time between the arrival of the task and the dispatch of the task. Here $N$ is total number of services in $S$.

$$
o b j_{1}=\sum_{\forall s_{i} \in S}\left(o_{i}^{t}-a_{i}^{t}\right) / N
$$

(O2) Average left-over charge: This objective describes the average left-over charge, which is the charge that remains in the battery after the flight.

$$
o b j_{2}=\sum_{\substack{\forall b_{j} \in B, \forall s_{i}, s_{i} \in b_{j} . S}}\left(\sum_{\forall s_{m} \in E\left(s_{i}, b_{j}\right)}\left(l_{m}^{c} k^{c}-l_{m}^{t} k^{t}\right)+l_{i}^{c} k^{c}-l_{i}^{t} k^{t}\right) / N
$$

(O3) Average charged waiting time: This objective describes the average charged waiting time of the tasks, which is represented as the time between the end of the charging operation and the dispatch time of the task.

$$
o b j_{3}=\sum_{\forall s_{i} \in S}\left(o_{i}^{t}-o_{i}^{c}-l_{i}^{c}\right) / N
$$

\section{EXPERIMENTS}

\section{A. Experiment Setup with Discrete-Event Simulator}

In order to evaluate the efficacy of the proposed algorithm, we use a custom developed simulator from [13]. The simulator incorporates all the detailed battery models presented in Section IV, such that we are able to correctly evaluate the gains of a battery management. The simulator is built around the event queue implemented as a priority queue, where the simulation events correspond to real-world events such as delivery service request arrival, drone departure/arrival, battery charging start, battery charging finish, and so on. When an event triggers, the corresponding callback function is called to perform related calculations such as battery SOC update, $\mathrm{SOH}$ update, drone status, etc. We emphasize that the gains are presented not in terms of the objective functions of the algorithm, but the actual $\mathrm{SOH}$ degradation obtained from the simulation. The overall evaluation flow is shown in Fig. 7. The discrete event simulator for drone delivery service implemented in $\mathrm{C}++$ communicates with an independent solver that embeds the proposed algorithm in Sections V-A and V-B implemented in MATLAB. The algorithm is invoked during the simulation run and solves the mapping, ordering, and scheduling problem repeatedly. As the algorithm requires knowledge of the service request times, we make an assumption that delivery requests are foreseeable for a certain time period in the future. This makes sense in that every delivery service require some amount of preparation time, e.g., locating the package in the storage, transporting the package to the drone platform, etc. Hence, we assume that the algorithm could find a time window for foreseeing the future.

\section{B. Results}

In this section, we show the amount of $\mathrm{SOH}$ degradation of the proposed method compared with two baseline techniques. The first baseline, random algorithm, assigns batteries randomly to delivery tasks as long as the energy constraints are met. The second one, capacity algorithm, searches through batteries, which has the least amount of energy meeting the requirement of a delivery task. Both algorithms charge batteries to its full capacity as soon as a delivery task is served. We use a task profile, where its flight distance follows a linear distribution between $0 \mathrm{~km}$ to $25 \mathrm{~km}$, and its number of services follows a Poisson distribution. We perform a simulation for a four year period serving delivery services of a million tasks with a battery set size of 75 . The battery set comprises 25 batteries of $226 \mathrm{Wh}$ capacity and 50 batteries of $452 \mathrm{Wh}$ capacity. The reason we use more larger batteries is that the flight distance distribution of tasks follows a linear distribution, and there are more long distance deliveries than short ones. The numbers of delivery services and batteries are smaller than what a real drone delivery business are likely to be, but it is enough to show the efficacy of the proposed method.

Fig. 9 and 10 show the detailed battery schedule for a day of the random algorithm and the proposed algorithm. Black boxes denote the charged-and-wait time, grey boxes denote discharge time, and white boxes denote the charge time, respectively. The most significant difference is that the former exhibits very long charge-and-wait times for a battery while the latter exhibits almost no charge and wait times. The batteries with an ID of less than 50 is used less than others because these are larger batteries only used for long distance flights. Fig. 8 shows the time-series comparison between the proposed algorithm and the capacity algorithm. From the SOC traces, it is noticeable that the average SOC is decreased in case of proposed algorithm, and hence, the capacity degradation is mitigated. The dense grey lines consisting of hundreds of charge/discharge cycles show SOC swing. For the proposed algorithm, the minimum SOC of each cycle is clamped to the minimum value, while it is clamped to the maximum value for the baseline. As a result, the capacity fade is much slower, making the battery usable well over the first 300 days. The proposed algorithm is capable of reducing the waiting time significantly as well, almost to half of the capacity baseline. The number of retired batteries over time is directly influenced by the rate of capacity fading, and there is significant reduction. Table I

\begin{tabular}{ccccc}
\hline Method & Elec. & Batt. & Sum & Wait time \\
\hline Random & $75,060.34$ & $52,199.24$ & $127,259.58$ & 271.27 \\
\hline Capacity & $73,479.21$ & $54,073.59$ & $127,552.80$ & 252.59 \\
\hline Proposed & $78,804.04$ & $15,889.47$ & $94,693.51$ & 108.32 \\
\hline \multicolumn{5}{c}{ TABLE 1} \\
\hline
\end{tabular}

OPERATING COST (IN USD) BREAKDOWN ANALYSIS AND PACKET WAITING TIME (S) FOR DIFFERENT ASSIGNMENT AND SCHEDULING TECHNIQUES.

provides the costs and packet waiting times. The proposed algorithm is capable of reducing the battery purchasing costs by significant proportion and reduces the sum of electricity cost and battery cost by more than $25 \%$ compared with the baselines, and packet waiting time by more than $50 \%$. The 

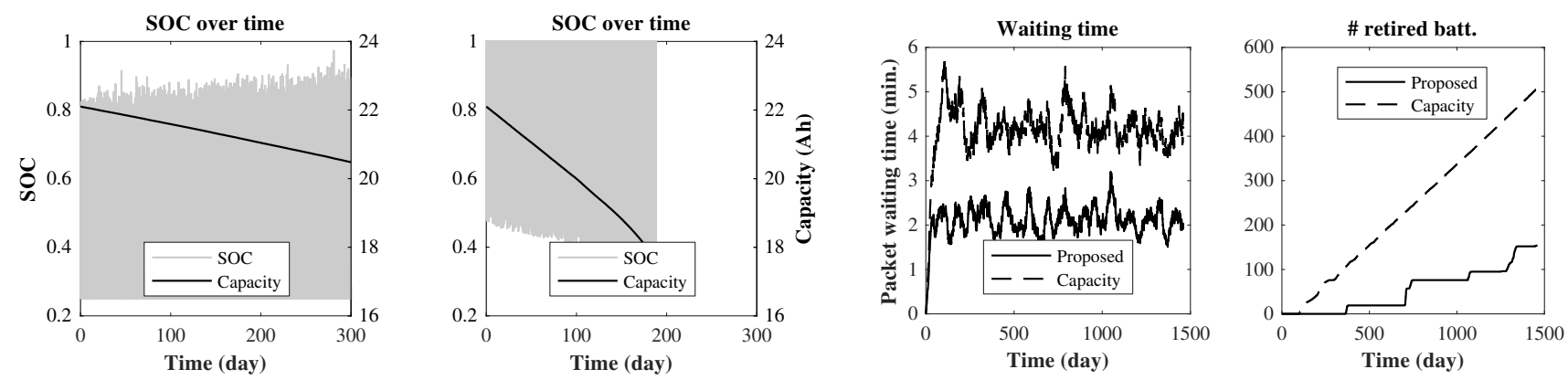

Fig. 8. SOC and capacity trace of a battery for proposed algorithm (leftmost), capacity baseline (second from left), packet waiting time (second from right), and number of retired batteries (rightmost).

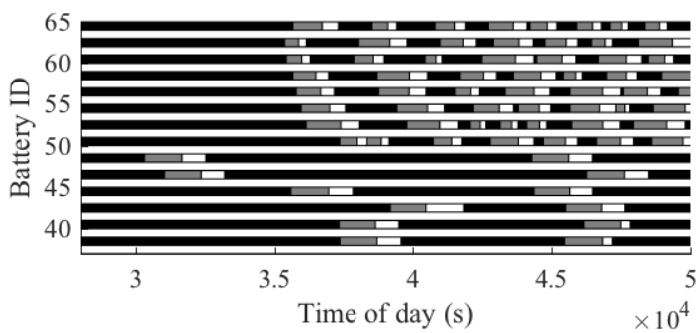

Fig. 9. Detailed battery schedule for a part of a day with charge-to-full scheme.

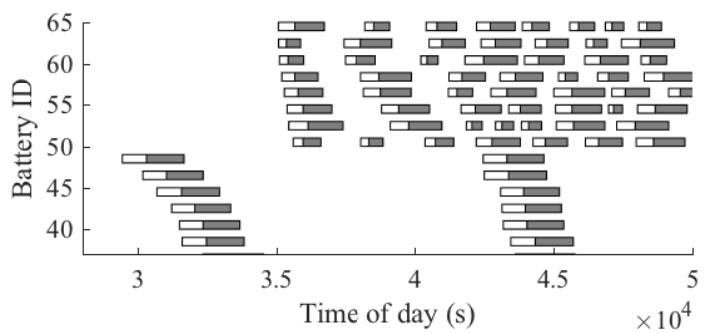

Fig. 10. Detailed battery schedule for a part of a day with the proposed scheme.

electricity cost is increased slightly for the proposed algorithm, which is one of the small blind spots in our algorithm. It is widely observed that the internal resistance is a function of SOC, where the value is increased when the SOC is low [6]. As our algorithm prefers to operate batteries in the low SOC region, it adversely affects the battery cycle efficiency, which results in slightly higher electricity cost. Nevertheless, huge gain from battery purchasing cost cancels out this effect. We acknowledge that the gain depends substantially on the delivery request profiles. For example, it is likely that drone flights will be forbidden during night times due to noise, safety, etc. If the length of this idle interval is longer, our algorithm shows larger gain, as it tries to maintain low battery SOC during the interval, while the baselines let the batteries wait fully-charged.

Lastly, the proposed algorithm provides leverage to exploit the trade-off relationship between the different objectives. For example, the weight values $(1,1,1)$ for objectives $(10)$ to $(12)$ provides, the packet waiting time of $69.57 \mathrm{~s}$, average surplus SOC of $7.57 \%$, while $(1,10,1)$, and $(1,20,1)$ provides $99.98 \mathrm{~s}$, $1.32 \%$ and $113.28 \mathrm{~s}, 0.34 \%$, respectively. This shows that as we put more emphasis on optimizing the average surplus charge, the gain accompanies sacrifice of packet waiting times.

\section{CONCLUDING REMARKS}

In this paper, we, for the first time, addressed the battery assignment and scheduling problem for drone delivery business under the objective of minimizing the $\mathrm{SOH}$ degradation.
We identify that the battery assignment affects the energy consumption of the delivery services, and the idle time between battery charge cycles have a significant impact on the $\mathrm{SOH}$ degradation of batteries. We formulate the battery assignment and scheduling problem as a two-stage problem where the mapping stage is solved by a heuristic and the scheduling stage is formulated as an ILP problem. The experimental results show that compared with the baseline policies, the proposed algorithm reduces the electricity and battery purchasing cost by $25 \%$ and packet waiting time by more than $50 \%$. Finding the truly global optimal solution for the $\mathrm{SOH}$ degradation minimization problem remains as a future work.

\section{ACKNOWLEDGEMENT}

This work is partially supported by the Deutsche Forschungsgemeinschaft (DFG) through the PARSEC project of TUM International Graduate School of Science and Engineering (IGSSE).

\section{REFERENCES}

[1] E. Ackerman. When drone delivery makes sense. IEEE Spectrum, 25, 2014.

[2] Airobotics. Airobotics Solution. http://www.airobotics.co.il/, 2017. [Online; accessed 10-Mar-2017].

[3] Amazon. Amazon Prime Air. https://www.amazon.com/ Amazon-Prime-Air/b?node=8037720011，2017. [Online; accessed 10-Mar-2017].

[4] Asylon. Introducing Dronehome. http://www.flyasylon.com/product/, 2016. [Online; accessed 10-Mar-2017].

[5] P.-J. Bristeau, F. Callou, D. Vissiere, N. Petit, et al. The navigation and control technology inside the ar. drone micro uav. In IFAC world congress, volume 18, pages 1477-1484, 2011.

[6] M. Chen and G. Rincon-Mora. Accurate electrical battery model capable of predicting runtime and I-V performance. IEEE Transactions on Energy Conversion, 21(2), 2006.

[7] R. D'Andrea. Guest editorial can drones deliver? IEEE Transactions on Automation Science and Engineering, 11(3):647-648, July 2014.

[8] J. Engel, J. Sturm, and D. Cremers. Camera-based navigation of a lowcost quadrocopter. In IEEE/RSJ IROS, 2012.

[9] M. O. Milhouse. Framework for autonomous delivery drones. In Proc. RIIT. ACM, 2015.

[10] A. Millner. Modeling lithium ion battery degradation in electric vehicles. In Proc. CITRES, pages 349-356, 2010.

[11] T. Motors. Battery swap. Fast Pack Swap Event http://www. teslamotors. com/batteryswap, 14, 2014.

[12] G. Ning, Z. Zhen, P. Wang, Y. Li, and H. Yin. Economic analysis on value chain of taxi fleet with battery-swapping mode using multiobjective genetic algorithm. Mathematical Problems in Engineering, 2012.

[13] S. Park, L. Zhang, and S. Chakraborty. Design space exploration of drone infrastructure for large-scale delivery services. In Proc. ICCAD. $\mathrm{ACM}, 2016$.

[14] T. Reddy. Linden's Handbook of Batteries, 4th Edition. McGraw-Hill Education, 2010.

[15] S. F. Schuster, T. Bach, E. Fleder, J. Müller, M. Brand, G. Sextl, and A. Jossen. Nonlinear aging characteristics of lithium-ion cells under different operational conditions. Journal of Energy Storage, 1, 2015.

[16] K. A. Suzuki, P. Kemper Filho, and J. R. Morrison. Automatic battery replacement system for uavs: Analysis and design. Journal of Intelligent \& Robotic Systems, 65(1-4):563-586, 2012. 